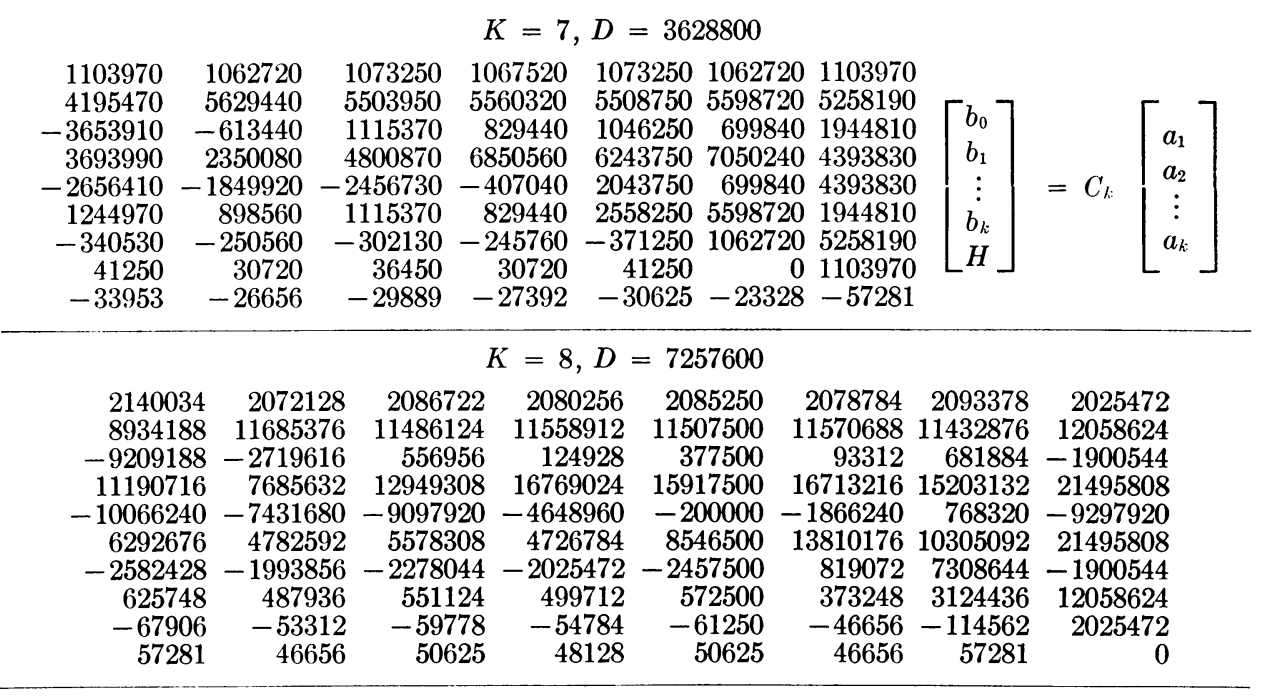

The Boeing Company

Renton, Washington

Now at

University of Alberta

Calgary, Alta, Canada

1. G. DAHLQUist, "Convergence and stability in the numerical integration of ordinary differential equations," Math. Scand., v. 4, 1956, p. 33-53.

2. T. E. Hull, \& A. C. R. NewBery, "Integration procedures which minimize propagated errors," J. Soc. Indust. Appl. Math., v. 9, 1961, p. 31-47.

3. - "Corrector formulas for multi-step integration methods," J. Soc. Indust. A ppl. Math., v. 10, 1962, p. 351-369.

4. H. A. Antosiewicz \& W. Gautschi, "Numerical methods in ordinary differential equations," A Survey of Numerical Analysis, Editor, J. Todd, McGraw-Hill Book Co., New York, 1962, p. 314-346.

\title{
On the Non-Existence of Fibonacci Squares
}

\section{By M. Wunderlich}

The Fibonacci sequence, $F_{n}$, is defined as follows:

$$
F_{1}=1 ; \quad F_{2}=1 ; \quad F_{n}=F_{n-2}+F_{n-1}
$$

for $n>2$.

A. P. Rollet [1] has posed the following problem. There are only three known Fibonacci numbers which are squares; $F_{1}=1, F_{2}=1$, and $F_{12}=144$. Are there any others? The purpose of this note is to announce that except for the known cases, $F_{n}$ cannot be a square for $n \leqq 1,000,000$, and to describe the computational method used to arrive at this result. The referee has kindly pointed out that the method used is somewhat analogous to familiar "exclusion" methods such as those described in [2].

Let $p$ be an arbitrary fixed prime number. With respect to this prime, denote by

Received November 20, 1962, revised April 10, 1963. 
$\bar{F}_{n}$ the least positive residue of $F_{n}$ modulo $p$. We will then refer to the sequence $\bar{F}_{1}$, $\bar{F}_{2}, \bar{F}_{3}, \cdots$ as the "reduced Fibonacci sequence." The mathematical basis for the method depends on the following elementary property of this sequence.

Theorem. The reduced Fibonacci sequence is purely periodic of period less than $p^{2}$, that is, there exists an $N \leqq p^{2}$ such that

$$
n \equiv m(N) \text { implies } \quad \bar{F}_{n}=\bar{F}_{m} .
$$

Proof. Consider the $p^{2}$ pairs of numbers

$$
\left(\bar{F}_{1}, \bar{F}_{2}\right), \quad\left(\bar{F}_{2}, \bar{F}_{3}\right), \quad \cdots\left(\bar{F}_{p^{2}}, \bar{F}_{p^{2}+1}\right)
$$

If each of the pairs is distinct from each other, then one of them must assume the value $(0,1)$ since there are only $p^{2}$ pairs of numbers modulo $p$. If $\left(\bar{F}_{t}, \bar{F}_{t+1}\right)=$ $(0,1)$, then it is clear that $\left(\bar{F}_{t+1}, \bar{F}_{t+2}\right)=(1,1)=\left(F_{1}, F_{2}\right)$, and the reduced sequence will be periodic with period $t$. If, on the other hand, two of the pairs in (2) are equal, say

$$
\begin{aligned}
\left(\bar{F}_{r}, \bar{F}_{r+1}\right) & =\left(\bar{F}_{m}, \bar{F}_{m+1}\right), & \text { where } r<m, \text { then we have } \\
\left(\bar{F}_{r-1}, \bar{F}_{r}\right) & =\left(\bar{F}_{m-1}, \bar{F}_{m}\right), & \text { and hence } \\
\left(\bar{F}_{r-2}, \bar{F}_{r-1}\right) & =\left(\bar{F}_{m-2}, \bar{F}_{m-1}\right), & \text { and eventually } \\
\left(\bar{F}_{1}, \bar{F}_{2}\right) & =\left(\bar{F}_{r-m}, \bar{F}_{r-m+1}\right)=(1,1) . &
\end{aligned}
$$

Hence the reduced sequence is periodic of period $r-m-1$.

Now let

$$
\bar{F}_{1}, \bar{F}_{2}, \cdots, \bar{F}_{M}
$$

be the first cycle of the sequence of reduced Fibonacci numbers, and let

$$
\bar{F}_{t_{1}}, \bar{F}_{t_{2}}, \cdots \bar{F}_{t_{k}}
$$

be the elements of $(3)$ which are equal to quadratic non-residues $\bmod p$. Then for $1 \leqq h \leqq k$, and $n \equiv t_{h}(M)$, we have from the theorem that $\bar{F}_{n}=\bar{F}_{t_{h}}$. Since $\bar{F}_{n}$ is a quadratic non-residue $\bmod p, F_{n}$ cannot be a square.

On the basis of probability considerations alone, it may be expected that approximately one half of the numbers in (3) are quadratic non-residues modulo $p$. Hence when applying this argument to a finite set of Fibonacci numbers it nay be expected to roughly halve the number of them which could be squares. Hence if one wishes to prove that the first 1,000,000 Fibonacci numbers contains only the three known squares, this argument would have to be repeated approximately $\log 1,000,000 / \log 2$ times, or approximately 20 times. Of course since this estimate is based on probability arguments alone, it is conceivable that it may take more repetitions to verify the result.

In order to program this sieve on the 709 I.B.M. computer, it was necessary to reserve one million storage locations in the machine, one for each of the first million Fibonacci numbers. Since for each of these numbers we had to determine whether it is not a square or could be a square, it was possible to use a single binary unit, a bit, for each of these locations. Therefore, we reserved one million consecu- 
tive bits to represent the first one million Fibonacci numbers and we adopted the following convention: If a one occupied the $n$th bit, this meant that $F_{n}$ could be a square. If a zero occupied the $n$th bit, $F_{n}$ could not be a square. These bits, which we shall call Fibonacci bits, were originally all set to contain ones.

In order to describe the program it is best to consider a special case. Let $p$ be the prime 7 . The first cycle of the sequence of reduced Fibonacci numbers are the 16 numbers

$$
1,1,2,3,5,1,6,0,6,6,5,4,2,6,1,0 .
$$

The quadratic non-residues of 7 are the numbers 3,5 , and 6 . If we rewrite (4) replacing the quadratic residues by ones and the quadratic non-residues by zeros and omitting the commas, we get

\section{1.}

In the program this expression was actually constructed as a binary number which was then used as a mask on the Fibonacci bits. Since there are 16 bits in $(5)$ the masking was executed as follows: If a zero occurred in the $n$th bit of $(5)$, zeros replaced all the $m$ th Fibonacci bits where $m \equiv n(16)$. If a one was in the $n$th bit of $(5)$, the $m$ th Fibonacci bits were unchanged. The 709 I.B.M. computer contains a single instruction which will perform such a logical operation on a single word of 36 bits. Therefore our mask could be performed with this instruction together with a series of shifting and storing instructions.

The entire program consisted of repeating this process with successive prime numbers beginning with 3 . After each repetition, the Fibonacci bits were tested to see if any ones remained and the program terminated when all the Fibonacci bits were zero, except for the three bits that corresponded to the known squares. This required the first 32 primes and was executed in six minutes. It should be pointed out that this method can only be used to demonstrate that a number is not a square, rather than proving that a number is a square. For example, if one of our Fibonacci numbers was of the form $1+\prod_{n<N} p_{n}$ where $p_{n}$ represents the $n$th prime number, its corresponding Fibonacci bit would remain a one for all primes less than $N$, and $N$ would not have to be too large for this test to become unreasonable even for a very fast computer.

The author would like to express his appreciation to Dr. W. Macintyre who provided him with the free use of the facilities at the Computation Center of the University of Colorado and to Dr. L. Moser who first suggested the problem to the author.

University of Colorado

Boulder, Colorado

1. A. P. Rollett, “Advanced Problem No. 5080," Amer. Math. Monthly, February, 1963.

2. J. V. Uspensky \& M. A. Heaslet, Elementary Number Theory, McGraw-Hill, New York, 1939, Chapter X, Sect. 19; Chapter XI, Sect. 8. 